\title{
Late hCG administration yields more good quality embryos and favors the overall IVF outcome
}

\author{
Pu Zhang*, Kjell Wånggren \\ Department of Women's and Children's Health, Uppsala University, Uppsala, Sweden \\ Email: ${ }^{*}$ zhangpu66@hotmail.com
}

Received 15 July 2012; revised 13 August 2012; accepted 22 August 2012

\begin{abstract}
Context: Optimal timing of hCG administration is a crucial step for successful IVF. Currently used standard hCG administration timing is not always practically possible due to weekends break or other reasons. Sometimes hCG needs to be administrated earlier or later than standard timing. Aim: To find out whether earlier or later hCG administration gives better IVF outcome. Setting and Design: A retrospective study on patients who underwent conventional IVF treatment. Methods and Material: Based on hCG timing, the patients were divided into three groups: the early hCG group where the hCG was given when less than three follicles $\geq 17 \mathrm{~mm}$; the standard hCG group where the hCG was given when three or more follicles $\geq 17 \mathrm{~mm}$; and the late hCG group where the hCG was given 1 to 3 days after the standard timing. The number of retrieved mature oocytes, the fertilization rate, the number of good quality embryos, the pregnancy rate and the live birth rate were compared among three groups. Statistical Analysis: $\mathrm{x}^{2}$ test, fisher exact test and Student t-test were used. Results: in total, 289 patients, 305 IVF cycles and 2784 oocytes were analyzed. The late hCG group has significantly larger number of MII oocytes, fertilized oocytes and good quality embryos per IVF cycle, when compared with the early hCG group. The fertilization rate, the pregnancy rate and the live birth rate per IVF cycle were similar among the three groups. Conclusion: Although the delayed administration of hCG did not favor IVF outcome per IVF cycle, the cumulative pregnancy rate is likely to be improved with consideration of higher yield of good quality embryos.
\end{abstract}

Keywords: hCG Timing; IVF; GnRH Agonist; GnRH Antagonist

${ }^{*}$ Corresponding author.

\section{INTRODUCTION}

Optimal timing of human chorionic gonadotrophin (hCG) administration is a crucial step for successful in-vitro fertilization (IVF). Currently most reproduction centers follow a commonly accepted guideline, that is, hCG is given when three or more follicles $\geq 17 \mathrm{~mm}$ are observed under ultrasound. This "standard" hCG administration timing, however, is not always practically possible. In practical work, because of weekend break or other reasons from the patients, sometimes hCG has to be administered one or two days earlier or later. If both options are available, which alternative is better, earlier or later? The answer is not yet clear. A few studies have looked at the effects of early or late hCG administration, but the results are controversial [1-6]. Most of these studies focus on the gonadotropin-releasing hormone $(\mathrm{GnRH})$ antagonist treatment $[1,3,4,6]$. In our reproduction center, we use more often GnRH agonist treatment. In this study, we compared early, late and standard hCG timing, and analyzed the effect of the different timing with respect to the number of retrieved mature oocytes, the fertilization rate, the number of good quality embryos, the pregnancy rate and the live birth rate.

\section{MATERIALS AND METHODS}

\subsection{Materials}

This retrospective study was carried out at our center for reproduction. The study was approved by the regional ethical review board. Dnr 2010/260.

289 patients who underwent conventional IVF treatment under the years 2008 and 2009 at our reproduction center were included in the study. 16 patients underwent two IVF treatments with the same or different protocols, both treatments were included in the study. In total 305 IVF cycles were analyzed. Inclusion criteria for patients were age $\leq 38$ years and $\mathrm{BMI}<30$. All intracytoplasmic sperm injections (ICSI) were excluded, because ICSI could artificially affect the fertilization rate. Two experi- 
enced doctors and five experienced embryologists worked under the period, following the same principles for all the treatments that minimized the artificial effects on the results.

\subsection{Clinical Treatments and Study Setting}

GnRH agonist treatment: Pituitary desensitization was achieved by down-regulation using GnRH agonist, (Suprecur, Sanofi-aventis AB Stockholm, Sweden; or Synarela, Pfizer AB, Stockholm, Sweden) from day 21 of the previous cycle. After at least 14 days of agonist administration, FSH (Puregon, Merch Sharp \& Dohme, Stockholm, Sweden; or Gonal-f, Merck AB, Stockholm, Sweden) stimulation was commenced. At the same time, the dose of the agonist decreased to half. Blood test on estradiol (E2) was taken after 5 - 6 days of stimulation and FSH dose was adjusted according to the E2 level. The first ultrasound scan was carried out after 8 - 9 days of stimulation and repeated when necessary.

GnRH antagonist treatment: On menstruation day 2 or day 3, patients start subcutant injection of FSH (Puregon, Merch Sharp \& Dohme, Stockholm, Sweden; or Gonal-f, Merck AB, Stockholm, Sweden). On stimulating day 6, commenced GnRH antagonist (Orgalutran, Merch Sharp \& Dohme, Stockholm, Sweden; or Cetrotide, Merck AB, Stockholm, Sweden). daily $0.25 \mathrm{mg}$ subcutaneously. On the same day, blood test on serum E2. Dose of FSH was adjusted depending on the E2 level. The first ultrasound scan was carried out after $7-8$ days of stimulation and repeated when necessary.

The time point of administration of hCG was decided at the last ultrasound scan. HCG (Pregnyl, Merch Sharp \& Dohme, Stockholm, Sweden) 10,000 IU was given to most of the patients. $5000 \mathrm{IU}$ was given when the risk of over stimulation was considered high.

Based on the hCG timing, the patients were divided into three groups: the early hCG group where the hCG was given when the number of big follicles $(\geq 17 \mathrm{~mm})$ is less than three; the standard hCG group where the hCG was given when the number of big follicles $(\geq 17 \mathrm{~mm})$ is three or more; and the late hCG group where the hCG was given 1 to 3 days later than the standard timing.

Oocyte retrieval was carried out 36 hours after hCG administration under transvaginal ultrasound guidance. All the follicles visible under ultrasound were punctured.

Conventional IVF was performed in all patients, including donor IVF. Embryo transfer (ET) were performed mainly on day 2. A small proportion of patients had ET on day 3. Only one embryo was transferred each time. The embryos that could be used for transferring or freezing were considered "good quality embryos".

Luteal phase was supported with vaginal Progesterone MIC (Apoteket Produktion \& Laboratorier AB, Stock- holm, Sweden) $400 \mathrm{mg} \times 3$ daily until the pregnancy was confirmed by ultrasound on week $7-8$.

\subsection{Statistical Analysis}

The total number of retrieved oocytes, the number of retrieved mature oocytes, the fertilization rate, the number of good quality embryos, the pregnancy rate and the live birth rate were compared among the three groups.

All the quantitative data are expressed as mean \pm standard deviation (SD).

$\mathrm{x}^{2}$ test was used to evaluate the difference in causes of infertility among the three groups, the ratio of $\mathrm{GnRH}$ agonist/antagonist, the ratio of day2/day3 (D2/D3) ET, the fertilization rate, the pregnancy rate, the live birth rate, the ratio of $\mathrm{MII} /$ total retrieved oocytes and the ratio of good quality embryos/fertilized oocytes. Fisher exact test was used when the frequency value was less than five. Student t-test was used to evaluate difference in maternal age, the duration of FSH stimulation, the thickness of uterus endometrium, the number of retrieved oocytes per cycle, the number of MII oocytes per cycle, the number of fertilized oocytes per cycle and the number of good quality embryos per cycle.

Two tails P-value $<0.05$ was taken to be significant.

\section{RESULTS}

In total, 289 patients and 305 IVF treatments are included in this study.

Table 1 shows the characteristics of all the patients. Except more agonist-treatments in the standard and the late $\mathrm{hCG}$ group when compared with the early group, no significant differences are seen among the three groups regarding patients' age, the causes of infertility, the embryo transferring timing, the duration of FSH stimulation or the thickness of uterus endometrium.

Table 2 summarizes the results of the comparison among the early, the standard and the late hCG groups. In total, 2784 oocytes are retrieved. Of the total retrieved oocytes, the late and the standard hCG group have a significantly higher proportion of MII oocytes than the early hCG group: $89.36 \%$ and $89.18 \%$ vs $82.73 \%$. Correspondingly, the late hCG group has a significantly larger number of MII oocytes per IVF cycle when compared with the early hCG group: 8.53 vs 6.57 . As the fertilization rate, the ratio of good quality embryos/ fertilized oocytes are similar between the three groups, the higher yield of MII oocytes in the late hCG group leads to significant larger number of fertilized oocyte and good quality embryos per cycle, when compared with the early group. The pregnancy rates are similar among the three groups. The live birth rate is lower in the early hCG group than in the standard and the late groups; $28.57 \%$ vs $40.96 \%$ and $40.11 \%$. However, the difference is not 
Table 1. Patients' characteristics in the study.

\begin{tabular}{ccccc}
\hline & Early hCG group & Standard hCG group & Late hCG group & P-value \\
\hline Maternal age (years) & $33.1 \pm 3.9$ & $32.9 \pm 4.1$ & $32.4 \pm 3.8$ & NS \\
Tubal factor & $5.7 \%(2)$ & $12.0 \%(10)$ & $9.1 \%(17)$ & NS \\
Endometriosis & $20.0 \%(7)$ & $8.4 \%(7)$ & $13.4 \%(25)$ & NS \\
PCO & $14.3 \%(5)$ & $9.6 \%(8)$ & $12.3 \%(23)$ & NS \\
Unexplained & $42.9 \%(15)$ & $54.2 \%(45)$ & $42.2 \%(79)$ & NS \\
Lesbian & $11.4 \%(4)$ & $3.6 \%(3)$ & $4.3 \%(8)$ & NS \\
Male factor & $5.7 \%(2)$ & $6.0 \%(5)$ & $11.2 \%(21)$ & NS \\
Other & 0 & $6.0 \%(5)$ & $7.5 \%(14)$ & NS \\
Total IVF cycles & 35 & 83 & 187 & - \\
GnRH agonist/GnRH antagonist cycles & $15 / 20$ & $61 / 22^{*}$ & $163 / 24^{\dagger}$ & ${ }^{*}=0.0028$ vs early \\
Duration of FSH stimulation (days) & $10.40 \pm 2.69$ & $10.45 \pm 1.80$ & $10.84 \pm 1.89$ & NS \\
Thickness of uterus endometrium (mm) & $10.16 \pm 1.79$ & $10.66 \pm 2.34$ & $10.20 \pm 2.12$ & $150 / 35 / 2(\mathrm{D} 5)$ \\
D2/D3 ET & $23 / 12$ & $67 / 16$ & $130 / 52 / 5$ & $\mathrm{NS}$
\end{tabular}

$\mathrm{PCO}=$ Polycystic ovary; ET = embryo transfer; NS = no significant.

Table 2. Comparison among different hCG timing groups.

\begin{tabular}{|c|c|c|c|c|}
\hline & Early hCG group & Standard hCG group & Late $\mathrm{hCG}$ group & P-value \\
\hline Total IVF cycles & 35 & 83 & 187 & - \\
\hline Total retrieved oocytes & 278 & 721 & 1785 & - \\
\hline Total No. of MII oocytes & 230 & 643 & 1595 & - \\
\hline Total No. of fertilized MII oocytes & 157 & 448 & 1150 & - \\
\hline Total No. of good quality embryo & 262 & 81 & 646 & - \\
\hline No. of retrieved oocytes per cycle & $7.94 \pm 4.76$ & $8.69 \pm 4.98$ & $9.55 \pm 5.31$ & NS \\
\hline No. of MII oocytes per cycle & $6.57 \pm 3.94$ & $7.75 \pm 4.74$ & $8.53 \pm 4.73^{*}$ & ${ }^{*}=0.022$ vs early \\
\hline No. of fertilized oocytes per cycle & $4.49 \pm 2.79$ & $5.40 \pm 3.93$ & $6.15 \pm 4.14^{*}$ & ${ }^{*}=0.024$ vs early \\
\hline No. of good quality embryos per cycle & $2.31 \pm 1.64$ & $3.16 \pm 2.48$ & $3.45 \pm 2.60^{*}$ & ${ }^{*}=0.013$ vs early \\
\hline $\mathrm{MII} /$ total retrieved oocytes & $82.73 \%$ & $89.18 \%{ }^{*}$ & $89.36 \%{ }^{\dagger}$ & ${ }^{*}=0.008$ vs early, ${ }^{\dagger}=0.002$ vs early \\
\hline Fertilization rate & $68.26 \%$ & $69.67 \%$ & $72.10 \%$ & NS \\
\hline Good quality embryo/fertilized oocytes & $51.59 \%$ & $58.48 \%$ & $56.17 \%$ & NS \\
\hline Pregnancy rate & $51.43 \%$ & $56.63 \%$ & $51.87 \%$ & NS \\
\hline Live birth rate & $28.57 \%$ & $40.96 \%$ & $40.11 \%$ & NS \\
\hline
\end{tabular}

statistically significant.

Table 3 illustrates the comparison among the early, the one-day and the two-days delayed groups. There is no significant difference between one-day and two-days delayed groups. Both one-day and two-days delayed groups have better results than the early group regarding the number of MII oocytes, the number of fertilized oocytes and the number of good quality embryos per cycle. However, the live birth rate is similar among three groups.

We compared also standard and two-days delayed group. The results show that the two-days delayed group 
Table 3. Comparison among early, one-day and two-day delayed hCG groups.

\begin{tabular}{|c|c|c|c|c|}
\hline & Early & One-day delayed & Two-days delayed & P-value \\
\hline Total cycles & 35 & 130 & 52 & - \\
\hline No. of MII oocytes & $6.57 \pm 3.94$ & $8.53 \pm 4.84^{*}$ & $8.81 \pm 4.57^{\dagger}$ & ${ }^{\dagger}=0.02 ;{ }^{*}=0.029$ \\
\hline No. of fertilized oocytes & $4.49 \pm 2.79$ & $5.98 \pm 4.19^{*}$ & $6.75 \pm 4.12^{\dagger}$ & $\begin{aligned} * & =0.049 \\
\dagger & =0.005\end{aligned}$ \\
\hline No. of good quality embryos & $2.31 \pm 1.64$ & $3.40 \pm 2.51^{*}$ & $3.75 \pm 2.89^{\dagger}$ & $\begin{aligned} * & =0.017 \\
\dagger & =0.009\end{aligned}$ \\
\hline Live birth rate & $28.57 \%$ & $37.69 \%$ & $48.08 \%$ & NS \\
\hline
\end{tabular}

NS = no significant; The numbers of the oocytes and the embryos, the live birth rate are per cycle; There is no significant difference between one-day and two-day delaying. All the P-values are against early hCG group.

has a tendency to give better IVF results with regard to the MII oocyte yield per cycle (8.81 vs 7.75$)$, the number of fertilized oocytes per cycle (6.75 vs 5.40$)$, the number of good quality embryo per cycles (3.75 vs 3.16$)$ and the live birth rates $(48.08 \%$ vs $40.96 \%)$. But the differences are not statistically significant.

\section{DISCUSSIONS}

The results of this study suggests that, no matter GnRH antagonist or agonist treatment, if administration of hCG switch is needed, it is better to delay hCG timing rather than to advance it. The delayed administration of hCG may not favor IVF outcome in a single cycle, but the cumulative pregnancy rate is likely to be improved with consideration of higher yield of mature oocytes, fertilized oocytes and good quality embryos per IVF cycle.

Dimitry et al. found that a 24 hours delay in $\mathrm{hCG}$ administration under agonist treatment hade a higher pregnancy rate when compared to standard timing [2]. In our study, we did not observe the increased pregnancy rate. However, taking into account the larger number of good quality embryos that have been frozen, one could expect that the cumulative pregnancy rate and live birth rate would be higher in the delayed hCG group if patients undergo several ETs. Our results are also in agreement with Tremellen et al., although they investigated GnRH antagonist treatments [6]. Their study showed that one-day delaying of hCG administration did not affect IVF live birth rate, but did increase both the number of retrieved oocytes and created embryos.

Earlier reported beneficial effects of a delayed $\mathrm{hCG}$ timing are limited to one day. Our results show that both one-day and two-day delaying are better than early hCG timing. Two-day delaying seems have more beneficial effects than one-day delaying although the statistic analysis did not show the significance. Kolibianakis' group reported in a large randomized prospective trial that a two-day delaying of hCG under GnRH antagonist treatment did not affect oocyte or embryo quality but was associated with a significantly lower ongoing pregnancy rate [3]. The lower pregnancy rate, according to the same research group, could be due to extreme endometrial advancement as a higher incidence of premature secretary changes were found on the day of oocyte retrieval after two-day delaying [4]. Two other groups found that premature elevated progesterone could impair implantation and live birth rate $[7,8]$. Chuang et al. found that prolonged stimulation, 13 days or more vs 10 - 12 days, was associated with decreased live birth rate [1]. Concerning the other studies' results and the relatively small number of patients in our study, we cannot ultimately conclude that two-day delaying is better than one-day delaying.

In principle, delayed hCG timing yields a greater amount of larger follicles, which in turn yields more mature oocytes with better developmental competence, according to a number of earlier studies [9-16]. The relationship between the follicle sizes and the developmental competence of the oocytes has been investigated by different research groups. They found that oocytes from larger follicles have higher fertilization rate, cleavage rate, and implantation/pregnancy rate [9-16]. The mechanism is not yet clear; it may be due to better cytoplasm maturation in the oocytes from the larger follicles. In our study, we did not follow follicles/oocytes one by one, so it is difficult to say if the good quality embryos are from those oocytes within larger follicles or not. However, we found that the number of good quality embryos had good correlation with the number of follicles larger than $15 \mathrm{~mm}$ at the $\mathrm{hCG}$ administration. This suggests that follicle size could be used as one of the predictors for IVF outcome. The emergency of 3D measurement of oocytes could help doctors make better predictions [17].

Among most of the IVF cycles, we notice a big drop from fertilized oocytes to good quality embryos, no matter it is on day 2 or day 3 . It seems that a majority $(83 \%-89 \%)$ of retrieved oocytes are mature and most $(68 \%-72 \%)$ of these mature oocytes can be fertilized, but only half $(52 \%-58 \%)$ of them develop further to embryos with good quality. To improve the final IVF outcome, more efforts are needed to optimize the development of the embryo after fertilization, for example, by 
changing the culture medium or lowering the osmolarity [18]. If we can achieve better development of embryo in vitro, we will have a much higher IVF success rate in the future.

In our study, we grouped the patients in respect of agonis/antagonist treatment. As showed in Table 1, we hade more antagonist cycles in early hCG group, and more agonist cycles in the standard and late hCG group. Concern about biased results may arise as some earlier studies showed that the agonist protocol gives the better oocyte yield and pregnancy rates $[19,20]$. However, most of studies do not show any significant difference between the two treatments [21,22] and the conception is now generally accepted. Therefore, we believe that the differences we observed among the three hCG groups are not biased by the different ratio of agonist/antagonist cycles among the groups.

In summary, our study together with other published data indicates that if the switching of hCG administration timing is needed, one-day delaying is better than early timing. To decide whether or not the current "standard" timing should be changed to one day later, larger randomized controlled trials are needed before a clear answer can be provided.

\section{REFERENCES}

[1] Chuang, M., Zapantis, A., Taylor, M., Jindal, S.K., NealPerry, G.S., Lieman, H.J. and Polotsky, A.J. (2010) Prolonged gonadotropin stimulation is associated with decreased ART success. Journal of Assisted Reproduction and Genetics, 27, 711-717. doi:10.1007/s10815-010-9476-6

[2] Dimitry, E.S., Oskarsson, T., Conaghan, J., Margara, R. and Winston, R.M. (1991) Beneficial effects of a $24 \mathrm{~h}$ delay in human chorionic gonadotrophin administration during in-vitro fertilization treatment cycles. Human Reproduction, 6, 944-946.

[3] Kolibianakis, E.M., Albano, C., Camus, M., Tournaye, H., Van Steirteghem, A.C. and Devroey, P. (2004) Prolongation of the follicular phase in in-vitro fertilization results in a lower ongoing pregnancy rate in cycles stimulated with recombinant follicle-stimulating hormone and gonadotropin-releasing hormone antagonists. Fertility and Sterility, 82, 102-107.

doi:10.1016/i.fertnstert.2004.01.027

[4] Kolibianakis, E.M., Bourgain, C., Papanikolaou, E.G., Camus, M., Tournaye, H., Van Steirteghem, A.C. and Devroey, P. (2005) Prolongation of follicular phase by delaying hCG administration results in a higher incidence of endometrial advancement on the day of oocyte retrieval in GnRH antagonist cycles. Human Reproduction, 20, 2453-2456. doi:10.1093/humrep/dei069

[5] Tan, S.L., Balen, A., El Hussein, E., Mills, C., Campbell, S., Yovich, J. and Jacobs, H.S. (1992) A prospective randomized study of the optimum timing of human chorionic gonadotropin administration after pituitary desensitiza- tion in in-vitro fertilization. Fertility and Sterility, 57, 1259-1264.

[6] Tremellen, K.P. and Lane, M. (2010) Avoidance of weekend oocyte retrievals during $\mathrm{GnRH}$ antagonist treatment by simple advancement or delay of hCG administration does not adversely affect IVF live birth outcomes. Human Reproductio, 25, 1219-1224. doi:10.1093/humrep/deq059

[7] Kilicdag, E.B., Haydardedeoglu, B., Cok, T., Hacivelioglu, S.O. and Bagis, T. (2010) Premature progesterone elevation impairs implantation and live birth rates in GnRH-agonist IVF/ICSI cycles. Archives of Gynecology and Obstetrics, 281, 747-752.

doi:10.1007/s00404-009-1248-0

[8] Bosch, E., Labarta, E., Crespo, J., Simon, C., Remohi, J., Jenkins, J. and Pellicer, A. (2010) Circulating progesterone levels and ongoing pregnancy rates in controlled ovarian stimulation cycles for in vitro fertilization: Analysis of over 4000 cycles. Human Reproduction, 25, 2092-2100. doi:10.1093/humrep/deq125

[9] Bergh, C., Broden, H., Lundin, K. and Hamberger, L. (1998) Comparison of fertilization, cleavage and pregnancy rates of oocytes from large and small follicles. Human Reproduction, 13, 1912-1915. doi:10.1093/humrep/13.7.1912

[10] Padhy, N., Latha, M., Sathya, B. and Varma, T.R. (2009) Antral follicle size in the downregulated cycle and its relation to in vitro fertilization outcome. Journal of Human Reproductive Sciences, 2, 68-71. doi:10.4103/0974-1208.57225

[11] Rosen, M.P., Shen, S., Dobson, A.T., Rinaudo, P.F., McCulloch, C.E. and Cedars, M.I. (2008) A quantitative assessment of follicle size on oocyte developmental competence. Fertility and Sterility, 90, 684-690. doi:10.1016/i.fertnstert.2007.02.011

[12] Saith, R.R., Srinivasan, A., Michie, D. and Sargent, I.L. (1998) Relationships between the developmental potential of human in-vitro fertilization embryos and features describing the embryo, oocyte and follicle. Human Reproduction Update, 4, 121-134. doi:10.1093/humupd/4.2.121

[13] Dubey, A.K., Wang, H.A., Duffy, P. and Penzias, A.S. (1995) The correlation between follicular measurements, oocyte morphology, and fertilization rates in an in vitro fertilization program. Fertility and Sterility, 64, 787-790.

[14] Miller, K.F., Goldberg, J.M. and Falcone, T. (1996) Follicle size and implantation of embryos from in vitro fertilization. Obstetrics \& Gynecology, 88, 583-586. doi:10.1016/0029-7844(96)00241-4

[15] Wittmaack, F.M., Kreger, D.O., Blasco, L., Tureck, R.W., Mastroianni Jr., L. and Lessey, B.A. (1994) Effect of follicular size on oocyte retrieval, fertilization, cleavage, and embryo quality in in vitro fertilization cycles: A 6-year data collection. Fertility and Sterility, 62, 1205-1210.

[16] Andersen, C.Y. (1993) Characteristics of human follicular fluid associated with successful conception after in vitro fertilization. Journal of Clinical Endocrinology \& $\mathrm{Me}$ tabolism, 77, 1227-1234. doi:10.1210/jc.77.5.1227

[17] Shmorgun, D., Hughes, E., Mohide, P. and Roberts, R. 
(2010) Prospective cohort study of three- versus two-dimensional ultrasound for prediction of oocyte maturity. Fertility and Sterility, 93, 1333-1337. doi:10.1016/i.fertnstert.2008.11.010

[18] Baltz, J.M. and Tartia, A.P. (2010) Cell volume regulation in oocytes and early embryos: Connecting physiology to successful culture media. Human Reproduction Update, 16, 166-176. doi:10.1093/humupd/dmp045

[19] Daya, S. (2000) Gonadotropin releasing hormone agonist protocols for pituitary desensitization in in vitro fertilization and gamete intrafallopian transfer cycles. Cochrane Database of Systematic Reviews, 1, CD001299.

[20] Muasher, S.J., Abdallah, R.T. and Hubayter, Z.R. (2006) Optimal stimulation protocols for in vitro fertilization.
Fertility and Sterility, 86, 267-273. doi:10.1016/j.fertnstert.2005.09.067

[21] Bodri, D., Sunkara, S.K. and Coomarasamy, A. (2011) Gonadotropin-releasing hormone agonists versus antagonists for controlled ovarian hyperstimulation in oocyte donors: A systematic review and meta-analysis. Fertility and Sterility, 95, 164-169. doi:10.1016/j.fertnstert.2010.06.068

[22] Devroey, P., Aboulghar, M., Garcia-Velasco, J., Griesinger, G., Humaidan, P., Kolibianakis, E., Ledger, W., Tomas, C. and Fauser, B.C. (2009) Improving the patient's experience of IVF/ICSI: A proposal for an ovarian stimulation protocol with GnRH antagonist co-treatment. Human Reproduction, 24, 764-774. doi:10.1093/humrep/den468 